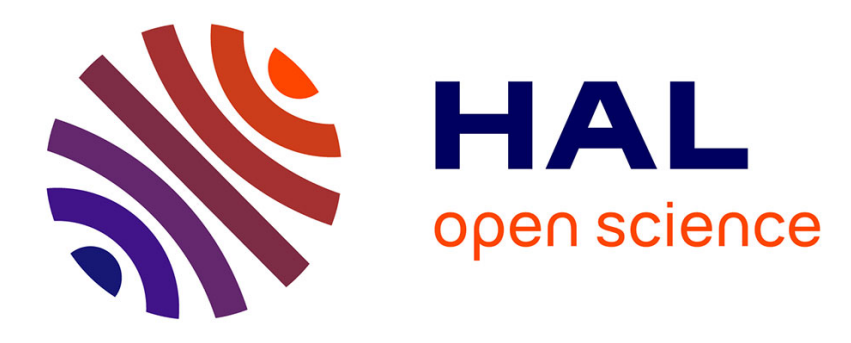

\title{
Modélisation et simulation d'un champ d'héliostats réfléchissant le rayonnement solaire direct vers une cible fixe
}

J.C. Hennet

\section{- To cite this version:}

J.C. Hennet. Modélisation et simulation d'un champ d'héliostats réfléchissant le rayonnement solaire direct vers une cible fixe. Revue de Physique Appliquée, 1983, 18 (5), pp.287-293. 10.1051/rphysap:01983001805028700 . jpa-00245093

\section{HAL Id: jpa-00245093 https://hal.science/jpa-00245093}

Submitted on 1 Jan 1983

HAL is a multi-disciplinary open access archive for the deposit and dissemination of scientific research documents, whether they are published or not. The documents may come from teaching and research institutions in France or abroad, or from public or private research centers.
L'archive ouverte pluridisciplinaire HAL, est destinée au dépôt et à la diffusion de documents scientifiques de niveau recherche, publiés ou non, émanant des établissements d'enseignement et de recherche français ou étrangers, des laboratoires publics ou privés. 


\title{
Modélisation et simulation d'un champ d'héliostats réfléchissant le rayonnement solaire direct vers une cible fixe
}

\author{
J. C. Hennet \\ Laboratoire d'Automatique et d'Analyse des Systèmes du C.N.R.S., \\ 7, avenue du Colonel Roche, 31400 Toulouse, France
}

(Reçu le 27 septembre 1982, révisé le 14 février 1983, accepté le 17 février 1983)

\begin{abstract}
Résumé. - Cet article décrit une modélisation analytique utilisée pour simuler et évaluer des champs d'héliostats focalisants.

Les calculs des densités de flux sont effectués pour chaque héliostat par intégration directe d'un produit de convolution simplifié. Ensuite, on évalue analytiquement les effets d'ombre, ce qui permet l'obtention de la carte globale de flux sur la cible par sommation des contributions.

Cette méthode est rapide et précise. Elle permet en outre de prendre en considération les erreurs de pointage et les défauts des surfaces réfléchissantes.
\end{abstract}

\begin{abstract}
This paper presents analytical models used to simulate and to evaluate fields of focusing heliostats. For each single heliostat, flux density calculations are performed through a direct integration of a simplified convolution product. Then, shadow effects are analytically computed and individual flux contributions are summed in order to get the global map of flux on the target.

This method is fast and accurate and allows for taking into account guidance errors and imperfections of reflecting surfaces.
\end{abstract}

1. Introduction. - La filière de conversion thermodynamique de l'énergie solaire par des centrales à tour a suscité ces dernières années de nombreux efforts de recherche, qui ont abouti en particulier à la réalisation de centrales expérimentales comme THEMIS ([6] et [7]) et SOLAR 1 aux Etats-Unis. Les progrès réalisés dans la conception de récepteurs solaires et les possibilités d'utilisation directe de la chaleur haute température permettent d'espérer parvenir au seuil de rentabilité de cette filière vers la fin du siècle, du moins pour les pays à fort ensoleillement direct. Le principal obstacle au développement de cette technologie est l'importance des investissements nécessités par la construction d'un champ d'héliostats et d'une tour. Pour parvenir à une meilleure conception de ce type de concentrateur, nous avons construit des méthodes de simulation et d'évaluation relativement rapides et précises. En ce qui concerne le calcul des flux réfléchis sur une cible fixe et le calcul des effets d'ombre entre héliostats, nous avons obtenu des expressions analytiques qui permettent d'alléger les calculs sur ordinateurs par rapport aux méthodes traditionnelles d'optique des cônes et de discrétisation. Les méthodes proposées permettent en outre de tenir compte aisément des défauts prévisibles des surfaces optiques et des imprécisions de pointage, ce qui permet de simuler correctement les comportements réels des héliostats.

\section{Calcul du flux réfléchi par un héliostat focalisant.}

- La densité de flux généré en un point de la surface d'ouverture du récepteur solaire par un héliostat réfléchissant le rayonnement solaire vers ce récepteur peut s'exprimer comme une intégrale double définie soit sur le disque solaire, soit sur la surface réfléchissante, soit sur la surface d'ouverture. Ces différentes approches ont été clairement décrites dans un article de F. W. Lipps [5]. Les méthodes couramment utilisées sont, soit la résolution numérique directe de l'intégrale double après discrétisation de la surface réfléchissante (méthode d'optique des cônes), soit le développement de l'intégrale, considérée comme une convolution de densités de probabilité, en polynômes d'Hermite à 2 dimensions (M. D. Walzel et al. [8]). Tandis que la première méthode exige des temps de calcul considérables, la seconde se révèle imprécise pour les centrales solaires à petite et moyenne échelle. Nous allons montrer que, sous quelques hypothèses simplificatrices, il est possible $\mathrm{d}_{\sim}$ résoudre analytiquement ce calcul intégral. 
2.1 IMAge PRINCIPALE D'Un HÉlIOSTAT. - Soit une surface réfléchissante de centre $H$ parfaitement pointée vers la cible. Le rayon incident principal issu du centre du soleil et atteignant le point $\mathrm{H}$ est réfléchi suivant un rayon principal de réflexion interceptant la cible au point $Z$. On définit le plan de calcul appelé plan image comme passant par $\mathrm{Z}$ et perpendiculaire au rayon principal de réflexion issu de $\mathrm{H}$. L'ensemble des rayons principaux de réflexion issus de tous les points de la surface réfléchissante intercepte le plan image suivant une surface appelée image principale de la surface réfléchissante.

Dans le cas où le rayon incident principal en $\mathrm{H}$ coïncide avec la normale à la surface réfléchissante en ce point, on sait que la surface qui permet une focalisation ponctuelle parfaite des rayons principaux de réflexion est le paraboloïde de révolution. En ce qui concerne les centrales à tour, le pointage des rayons réfléchis par les héliostats vers la chaudière ne permet pas de conserver l'axe de la normale à l'héliostat en son centre pointé vers le soleil. On peut alors choisir des surfaces réfléchissantes sphériques, et non plus paraboliques, dont le rayon de courbure est choisi tel que les facteurs de focalisation tangentielle et sagittale soient égaux. Le rayon vaut alors (HELIOS [2]) :

$$
R=2 \mathrm{HZ} \text {. }
$$

A titre d'illustration, on a représenté schématiquement sur la figure 1 un héliostat focalisant de contour rectangulaire et son image principale.

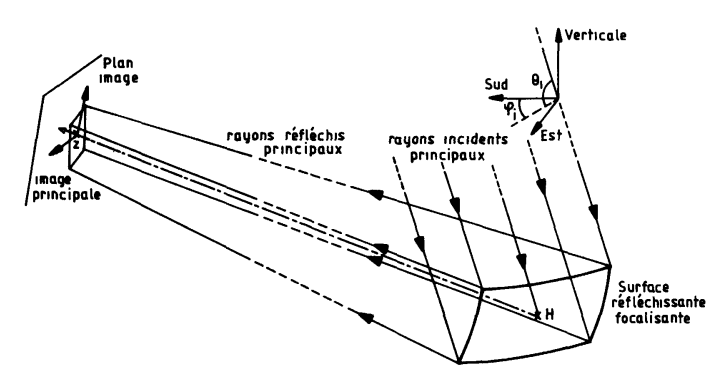

Fig. 1. - Image principale d'un héliostat.

[The principal image of a heliostat.]

2.2 CARACTÉRISATION DU RAYONNEMENT SOLAIRE DIRECT. - Jusqu'à présent nous avons caractérisé le fayonnement solaire incident par la seule direction du rayon principal, issu du centre du soleil. Or le soleil n'est pas une source ponctuelle d'énergie. Les rayons incidents en un point quelconque se répartissent dans un cône d'angle solide :

$$
W=\pi \alpha_{\mathrm{S}}^{2}
$$

où $\alpha_{S}$ est le rayon apparent du soleil $\left(\alpha_{S} \simeq 0,0046 \mathrm{rad}\right.$.). Soit un rayon incident faisant un angle $\alpha$ avec la direction du rayon principal. On note $I(\alpha)$ l'intensité du rayonnement solaire dans la direction caractérisée par l'angle $\alpha$. La décroissance de $I(\alpha)$ en fonction de $\alpha$ (pour $0 \leqslant \alpha \leqslant \alpha_{\mathrm{S}}$ ) traduit le phénomène d'assombrissement radial, pour lequel plusieurs modèles empiriques ont été élaborés. Nous retenons le modèle suivant, expérimenté par M. D. Walzel [8] : pour $0 \leqslant \alpha \leqslant \alpha_{\mathrm{s}}$ :

$$
I(\alpha)=I_{0}\left[1-\lambda\left(\frac{\alpha}{\alpha_{s}}\right)^{4}\right]
$$

avec $\lambda \simeq 0,5138$

L'intensité $I_{0}$ du rayonnement dans la direction principale est reliée à l'éclairement normal au sol, $E_{\text {n }}$, par la relation approchée suivante :

$$
I_{0} \simeq \frac{E_{\mathrm{n}}}{W(1-\lambda / 3)}
$$

2.3 EXPRESSIONS DE LA DENSITÉ DE FLUX SUR LE PLAN IMAGE. - Considérons un élément de surface $\mathrm{d} S \mathrm{du}$ miroir, centré au point $\mathrm{M}$, et un élément de surface $\mathrm{d} S^{\prime}$ du plan image, centré au point $Q$. Soit $\alpha$ l'angle de la direction de $\mathrm{MQ}$ avec la direction du rayon principal de réflexion en $\mathbf{M}$.

D'après la relation de Lambert, la contribution de $\mathrm{d} S$ à l'éclairement au point $\mathrm{Q}$, de coordonnées $(X, Y)$ dans le plan image, vaut :

- si $\alpha>\alpha_{\mathrm{s}}$

$$
\mathrm{d} F(X, Y)=0,
$$

- si $0 \leqslant \alpha \leqslant \alpha_{\mathbf{S}}$ (cas représenté à la figure 2)

$$
\mathrm{d} F(X, Y)=\rho \frac{I(\alpha) \cos \gamma \cos \alpha}{\mathrm{MQ}^{2}} \mathrm{~d} S
$$

avec $\rho$ réflectivité du miroir en $M$, $\gamma$ angle de MQ avec la normale au miroir en $\mathbf{M}$.

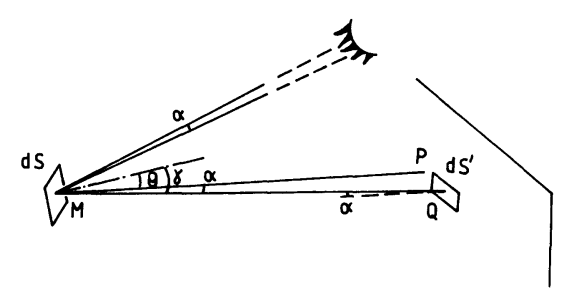

Fig. 2. - Réflexion du flux solaire par un élément de miroir. [Reflection of the solar flux by an elementary mirror.]

Soit $\mathrm{P}\left(X_{\mathrm{P}}, Y_{\mathrm{P}}\right)$ le point d'intersection du rayon principal de réflexion en $\mathrm{M}$ avec le plan image.

Soit $\theta$ l'angle de MP avec la normale à $\mathrm{d} S$ et soit $d$ la distance MP.

On a

$$
\alpha=\operatorname{Arctg}\left(\frac{\sqrt{\left(X-X_{\mathrm{P}}\right)^{2}+\left(Y-Y_{\mathrm{p}}\right)^{2}}}{d}\right) .
$$


Comme $\alpha$ est un angle très petit, on peut simplifier l'expression de $\mathrm{d} F(X, Y)$ :

$$
\mathrm{d} F(X, Y) \simeq \rho \frac{I(\alpha) \cos \theta}{d^{2}} \mathrm{~d} S
$$

ce qui peut ainsi s'écrire :

$$
\mathrm{d} F(X, Y)=\rho I(\alpha)\left(\frac{\mathrm{d} S}{\mathrm{~d} S^{\prime}}\right) \frac{\cos \theta}{d^{2}} \mathrm{~d} S^{\prime} .
$$

On peut repérer la direction de MQ par rapport à MP par deux angles, l'angle d'écartement $\alpha$ et un angle $\varphi$ de rotation autour de l'axe MP $(0 \leqslant \varphi \leqslant 2 \pi)$. Le terme $\left(\mathrm{d} S^{\prime} / \mathrm{d} S\right)$ est la valeur au point $\mathrm{Q}$ du jacobien de la transformation ponctuelle $T_{\alpha \varphi}$ caractéristique des rayons réfléchis de direction $(\alpha, \varphi)$ par rapport aux rayons réfléchis principaux. $\mathrm{Si}$ aucun des pseudofoyers de la transformation ponctuelle $T_{\alpha \varphi}$ n'est situé sur le plan image, ce qui est toujours le cas physiquement, la transformation ponctuelle $\mathrm{T}_{\alpha \varphi}$ est biunivoque et la valeur du jacobien est non nulle. Le terme $\left(\mathrm{d} S / \mathrm{d} S^{\prime}\right)$ correspond alors à l'inverse du jacobien au point $\mathrm{Q}$.

Etant données les faibles dimensions de l'image par rapport à la distance héliostat-tour, on peut assimiler l'image de l'héliostat par $T_{\alpha \varphi}\left(\operatorname{avec} 0 \leqslant \alpha \leqslant \alpha_{S}\right)$, à l'image principale (obtenue pour la transformation ponctuelle $T_{00}$ ), translatée dans le plan image du vecteur de composantes

$$
\mid \begin{aligned}
& x=d \cos \varphi \operatorname{tg} \alpha \\
& y=d \sin \varphi \operatorname{tg} \alpha .
\end{aligned}
$$

La valeur de $\left(\mathrm{d} S / \mathrm{d} S^{\prime}\right)$ au point $\mathrm{Q}$ vaut alors :

$$
\left(\frac{\mathrm{d} S}{\mathrm{~d} S^{\prime}}\right) \simeq C(X-x, Y-y)
$$

où $C(\xi, \eta)$ est l'inverse du jacobien de la transformation $\mathrm{T}_{00}$ au point $(\xi, \eta)$ si celui-ci appartient à l'image principale. Sinon, $C(\xi, \eta)=0$.

Comme $\alpha=\operatorname{Arctg} \frac{\sqrt{x^{2}+y^{2}}}{d}, I(\alpha)$ peut être représenté comme une fonction $G$ de $(x, y)$ définie de la façon suivante :

- $\mathrm{Si} \sqrt{x^{2}+y^{2}} \leqslant d \operatorname{tg} \alpha_{\mathrm{S}}$ (intérieur du disque $\mathfrak{D}$ de rayon $d \operatorname{tg} \alpha_{\mathrm{s}}$ )

$$
G(x, y)=I(\alpha) \text { pour } \alpha=\operatorname{Arctg} \frac{\sqrt{x^{2}+y^{2}}}{d} .
$$

- $\operatorname{Si} \sqrt{x^{2}+y^{2}}>d \operatorname{tg} \alpha_{\mathrm{S}}$ (extérieur du disque $\mathfrak{D}$ )

$$
G(x, y)=0 \text {. }
$$

Alors, la densité de flux au point courant du plan image vaut :

$$
\begin{aligned}
F(X, Y)=\rho \frac{\cos \theta}{d^{2}} \int_{-\infty}^{+\infty} & \int_{-\infty}^{+\infty} c(x, y) \times \\
& \times G(X-x, Y-y) \mathrm{d} x \mathrm{~d} y .
\end{aligned}
$$

Sous cette forme, l'intégrale apparaît clairement comme la convolution sur le plan image de la distribution de concentration géométrique de l'image principale avec l'intensité d'éclairement sur l'image du disque solaire. La densité de flux peut aussi s'écrire de façon équivalente :

$$
\begin{aligned}
F(X, Y)=\rho \frac{\cos \theta}{d^{2}} \int_{-\infty}^{+\infty} & \int_{-\infty}^{+\infty} C(x, y) \times \\
& \times G(X-x, Y-y) \mathrm{d} x \mathrm{~d} y .
\end{aligned}
$$

2.4 RÉSOLUTION SIMPLIFIÉE DU PRODUIT DE CONVOLUTION. - Pour résoudre de façon approchée l'intégrale de convolution, nous pouvons utiliser les hypothèses simplificatrices suivantes :

- Le jacobien de la transformation ponctuelle $\mathrm{T}_{00}$ est supposé constant en chaque point de l'image principale, et donc :

$\forall(\xi, \eta) \in$ image principale

$$
C(\xi, \eta)=C .
$$

$\forall(\xi, \eta) \notin$ image principale

$$
C(\xi, \eta)=0 \text {. }
$$

- La transformée par $\mathrm{T}_{00}$ d'une arête du miroir est supposée rectiligne. Elle est caractérisée simplement par la projection des deux sommets qu'elle joint. L'image principale est alors un quadrilatère noté J.

Sous ces hypothèses, la densité de flux au point $(X, Y)$ s'écrit :

$$
F(X, Y)=\rho \frac{\cos \theta}{d^{2}} C \iint_{J_{\cap D}} G(X-x, Y-y) d x d y
$$

Toutes les configurations d'intersection entre le quadrilatère $\mathrm{J}$ et le disque $\mathrm{D}$ peuvent être décomposées en 3 configurations de base pour lesquelles on sait calculer l'intégrale.

a) Le disque $\mathrm{D}$ est inscrit dans le quadrilatère $\mathrm{J}$.

Alors, le domaine d'intégration est le disque $\mathrm{D}$, et donc :

$$
F(X, Y)=F_{\mathrm{MAX}}=\rho \frac{\cos \theta}{d^{2}} C \varphi_{0}
$$

avec

$$
\begin{aligned}
\varphi_{0} & =2 \pi I_{0} \int_{0}^{R}\left(1-\lambda \frac{r^{4}}{R^{4}}\right) r \mathrm{~d} r \\
R & =d \operatorname{tg} \alpha_{\mathrm{S}}
\end{aligned}
$$

d'où

$$
\varphi_{0} \simeq E_{\mathrm{n}}
$$


b) Intersection du type arête simple.

Ce cas est représenté sur la figure 3.

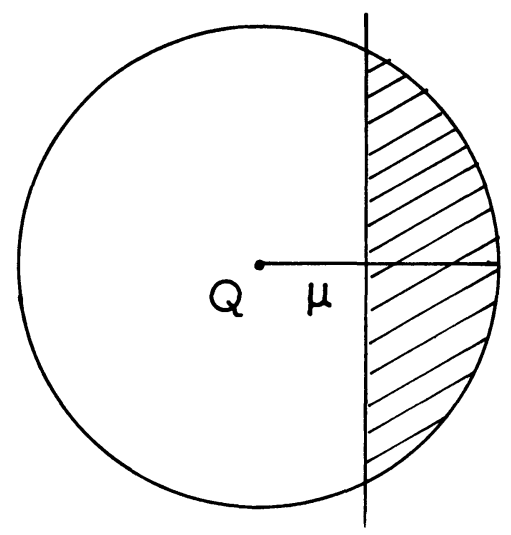

Fig. 3. - Intersection du type arête simple.

[Simple edge type intersection.]

On veut calculer l'intégrale sur l'aire hachurée :

$$
F(X, Y)=\rho \frac{\cos \theta}{d^{2}} C \varphi_{1}
$$

avec

$$
\varphi_{1}=\int_{\mu}^{R} 2 r \operatorname{Arccos}\left(\frac{\mu}{r}\right) I_{0}\left(1-\frac{\lambda r^{4}}{R^{4}}\right) \mathrm{d} r .
$$

On obtient, après intégration par parties :

$$
\begin{aligned}
\varphi_{1}=I_{0}\left[\left(1-\frac{\lambda}{3}\right)\right. & R^{2} \operatorname{Arccos} \frac{\mu}{R}-\mu \sqrt{R^{2}-\mu^{2}} \times \\
& \left.\times\left(\frac{3 R^{4}+4 \mu^{2} R^{2}+8 \mu^{4}}{90}+1\right)\right] .
\end{aligned}
$$

c) Un sommet de l'image principale est intérieur au disque.

Soit la nouvelle aire hachurée représentée sur la figure 4.

$$
K=\sqrt{R^{2}-v^{2} \sin ^{2} \omega}\left(3 R^{4}+4 R^{2} v^{2} \sin ^{2} \omega+8 v^{4} \sin ^{4} \omega\right)-v^{5} \cos \omega\left(15-20 \cos ^{2} \omega+8 \cos ^{4} \omega\right) .
$$

Une énumération de tous les cas de figures possibles serait fastidieuse. Nous nous sommes contentés, dans le mémoire [3], de présenter l'algorithme qui permet de calculer pour tous les points du plan image la valeur de la densité de flux à partir des expressions $\varphi_{0}, \varphi_{1}$ et $\varphi_{2}$.

3. Etablissement des cartes de flux pour un champ d'héliostats. - 3.1 CAS D'UN HÉLIOSTAT ISOLÉ DANS LE CHAMP. IMPRÉCISION DE POINTAGE ET DÉFAUTS OPTIQUES. - A partir du calcul des densités de flux sur le plan image, on peut aisément déterminer les

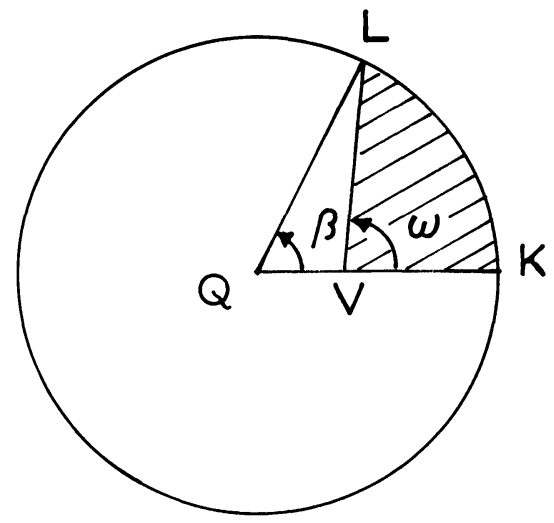

Fig. 4. - Intersection du type arête brisée.

[Broken edge type intersection.]

Cette aire est limitée par le prolongement VK du segment joignant $\mathrm{Q}$ au sommet $\mathrm{V}$ et par la portion d'arête orientée VL faisant un angle $\omega$ avec VK.

On suppose $0 \leqslant \omega \leqslant \pi / 2$, et l'on note $v=\overline{\mathrm{QV}}$ avec $0 \leqslant v \leqslant R$.

On définit l'angle $\beta$ comme l'angle $(\mathrm{QV}, \mathrm{QL})$. On a :

$$
\beta=\omega-\arcsin \left(\frac{v}{R} \sin \omega\right) \text {. }
$$

L'intégrale vaut :

$$
F(X, Y)=\rho \frac{\cos \theta}{d^{2}} C \varphi_{2}
$$

avec

$\varphi_{2}=\int_{v}^{R} I_{0}\left(1-\lambda \frac{r^{4}}{R^{4}}\right)\left(\omega-\operatorname{Arcsin}\left(\frac{v}{r} \sin \omega\right)\right) r \mathrm{~d} r$.

Par intégration par parties, on obtient

$$
\varphi_{2}=I_{0}\left[\frac{R^{2} \beta}{2}(1-\lambda / 3)-v\left(\frac{R \sin \beta}{2}-\lambda \frac{K \sin \omega}{90 R^{4}}\right)\right]
$$

avec

densités de flux reçu directement par la surface d'ouverture et par les parois de la chaudière. Il suffit en effet de projeter le point éclairé sur le plan image suivant la direction moyenne des rayons solaires réfléchis par l'héliostat. Dans le cas où la cible est plane, de contour rectangulaire, inclinée d'un angle $\theta_{c}$ par rapport à la verticale, on peut repérer un point courant $R$ du plan cible dans un repère lié à ce plan. Soient $\left(X_{\mathrm{R}}, Y_{\mathrm{R}}\right)$ les coordonnées de $\mathrm{R}$ dans ce repère. La projection de $R$ sur le plan image suivant le vecteur réfléchi principal d'angles $\left(\theta_{\mathrm{r}}, \varphi_{\mathrm{r}}\right)$ admet, dans le repère associé lié au plan image, les coordonnées $(X, Y)$ telles que : 


$$
\left(\begin{array}{l}
X \\
Y
\end{array}\right)=\left(\begin{array}{lc}
\cos \varphi_{\mathrm{r}} & -\sin \theta_{\mathrm{c}} \sin \varphi_{\mathrm{r}} \\
\sin \theta_{\mathrm{r}} \sin \varphi_{\mathrm{r}} & \cos \theta_{\mathrm{c}} \cos \theta_{\mathrm{r}}+\sin \theta_{\mathrm{c}} \sin \theta_{\mathrm{r}} \sin \varphi_{\mathrm{r}}
\end{array}\right)\left(\begin{array}{l}
X_{\mathrm{R}} \\
Y_{\mathrm{R}}
\end{array}\right)
$$

Ainsi, la carte de flux sur la cible peut être calculée très rapidement (environ $2 \mathrm{~s}$ sur IBM 3033) et avec une bonne précision, tandis que les méthodes classiques d'optique des cônes (P. Carraud et A. Giraud [1], C. Mersier [9]) ne convergent que lentement à mesure que le maillage de la surface réfléchissante devient plus serré.

Pour un héliostat parfaitement pointé, le rayon réfléchi principal en son centre intercepte la cible au point $Z$. Si le pointage est entaché d'erreur, le point d'intersection sur le plan image est décalé d'une distance $d \operatorname{tg} \varepsilon$, où $\varepsilon$ est l'angle d'écart sur le rayon réfléchi principal. Cet angle étant supposé relativement faible et la géométrie de l'héliostat restant la même, l'image principale de l'héliostat dépointé peut être assimilée à l'image principale sans dépointage translatée sur le plan image d'un vecteur de direction aléatoire et de norme $d \operatorname{tg} \varepsilon$. Dans les cas de forts dépointages, en particulier pour des dépointages volontaires, il convient de définir un nouveau plan image et une nouvelle image principale correspondant à la nouvelle cible.

Comme l'a montré R. O. Hughes [4] dans le cas des paraboloïdes réfléchissants orientables, il est nécessaire de traiter séparément les imprécisions de pointage et les défauts optiques des surfaces réfléchissantes. L'irrégularité d'une surface réfléchissante focalisante tend à diminuer son pouvoir concentrateur. Si un élément de surface réfléchissante a une orientation différente de celle qu'il devrait avoir, les rayons réfléchis qu'il émet s'écartent de la direction du rayon réfléchi principal théorique. En moyenne, tout se passe comme si l'angle d'ouverture des cônes de rayons réfléchis était plus grand. Pour simuler ce phénomène de façon simple, il suffit de reporter les écarts moyens du rayon réfléchi sur le rayonnement incident en élargissant le cône des rayons solaires incidents, pour un même bilan global de flux reçu par le miroir. Si l'on suppose un écart moyen $\bar{\delta}$ sur les normales aux facettes, on simulera cet effet en remplaçant $\alpha_{s}$ par $\alpha_{S}+2 \bar{\delta}$.

3.2 CALCUl DES EFFETS D'OMBRE ENTRE HÉLIOSTATS. - Certaines zones du site présentent, par leur position vis-à-vis de la chaudière, de meilleurs rendements optiques. Il est donc souhaitable d'y implanter un nombre relativement important d'héliostats. On est cependant limité par des contraintes mécaniques de débattement entre héliostats et l'on doit chercher un compromis entre l'utilisation intensive des zones à fort rendement et l'accroissement des ombres et des masquages entre héliostats lorsque la distance entre eux diminue.

On dit que l'héliostat $\mathscr{H}$ est ombré par l'héliostat $\mathfrak{H}^{\prime}$ lorsqu'une partie du rayonnement incident devant éclairer l'héliostat $\mathscr{H}$ est préalablement interceptée par l'héliostat $\mathcal{H}^{\prime}$.

De façon analogue, il y a masquage de l'héliostat $\nVdash e$ par l'héliostat $\mathfrak{J}^{\prime}$ lorsque certains rayons réfléchis par l'héliostat $H^{C}$ sont interceptés par la face arrière de l'héliostat $\mathcal{H}^{\prime}$.

L'effet d'ombre total de $X^{\prime}$ sur $\mathcal{H}$ est la conjonction de ces deux phénomènes d'ombre et de masquage de $\mathcal{H}^{\prime}$ sur $\mathcal{H}$. La surface non efficace de l'héliostat $\mathcal{H}$ est l'union de la surface ombrée et de la surface masquée.

Les méthodes de calcul des effets d'ombre habituellement utilisées sont de deux types :

- soit chaque zone du champ d'héliostats est considérée globalement et les effets d'ombre de cette zone sont caractérisés par leur valeur moyenne approchée (P. Courrège [10]);

- soit le contour exact de chaque héliostat est déterminé par projections géométriques des héliostats voisins (P. Carraud et A. Giraud [1]).

La taille relativement réduite des champs d'héliostats que nous étudions incite à chercher un compromis entre ces deux approches. Pour obtenir une sensibilité suffisante du calcul de flux à la position réelle des héliostats, nous particularisons chaque héliostat du champ. Mais pour ne pas trop accroître les tâches de calcul, la méthode analytique de calcul des effets d'ombres que nous proposons reste globale au niveau de chaque héliostat.

Pour calculer l'ombre et le masquage de $\mathfrak{H}^{\prime}$ sur $\mathfrak{H}$, on projette le contour de $\mathcal{H}^{\prime}$ sur le plan tangent à $J$ en son centre suivant respectivement la direction du rayon incident principal, $i$, et du rayon réfléchi principal, $r$. La projection de $\mathscr{H}^{\prime}$ sur $\mathscr{H}$ est assimilée à un rectangle de largeur $L_{\mathrm{c}}$ et de hauteur $H_{\mathrm{c}}$ dans le repère lié à $\nVdash(F i g .5)$. Ce repère est défini par la normale à $\mathcal{H}$ en son centre, de vecteur directeur

$$
\mathbf{e}_{1}=\left(\begin{array}{cc}
\cos \varphi_{\mathrm{n}} & \cos \theta_{\mathrm{n}} \\
\sin \varphi_{\mathrm{n}} & \cos \theta_{\mathrm{n}} \\
\sin \theta_{\mathrm{n}} &
\end{array}\right)
$$

La largeur cachée $L_{\mathrm{c}}$ est mesurée sur l'axe de vecteur directeur

$$
\mathbf{e}_{2}=\left(\begin{array}{c}
-\sin \varphi_{\mathrm{n}} \\
\cos \varphi_{\mathrm{n}} \\
0
\end{array}\right)
$$

La hauteur cachée est mesurée sur l'axe de vecteur directeur

$$
\mathbf{e}_{3}=\left(\begin{array}{cc}
-\cos \varphi_{\mathrm{n}} & \sin \theta_{\mathrm{n}} \\
-\sin \varphi_{\mathrm{n}} & \sin \theta_{\mathrm{n}} \\
\cos \theta_{\mathrm{n}} &
\end{array}\right)
$$




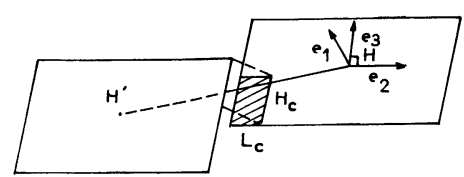

Fig. 5. - Projection de l'héliostat $\mathfrak{H}^{\prime}$ sur l'héliostat $\mathfrak{X}$.

[Projection of heliostat $\mathcal{H}^{\prime}$ onto heliostat $\mathcal{H}$.]

Dans le repère $\left(\mathrm{H}, \mathbf{e}_{1}, \mathbf{e}_{2}, \mathbf{e}_{3}\right)$, le vecteur de projection (i ou r) est caractérisé par les angles $\left(\theta_{\mathrm{P}}^{\prime}, \varphi_{\mathrm{P}}^{\prime}\right)$, et le vecteur des centres $H, H^{\prime}$ par sa norme, $s$, et par les angles $\left(\theta^{\prime}, \varphi^{\prime}\right)$. Le vecteur normal à l'héliostat $\mathcal{H}^{\prime}$ en son centre $\mathrm{H}^{\prime}$ n'est pas supposé égal à $\mathbf{e}_{1}$. Il est caractérisé dans le repère $\left(\mathrm{H}, \mathbf{e}_{1}, \mathbf{e}_{2}, \mathbf{e}_{3}\right)$ par les angles $\left(\theta_{\mathrm{n}}^{\prime}, \varphi_{\mathrm{n}}^{\prime}\right)$.

La largeur cachée est calculée en projection sur $\left(H, \mathbf{e}_{1}, \mathbf{e}_{2}\right)$, la hauteur cachée en projection sur $(H$, $\left.\mathbf{e}_{1}, \mathbf{e}_{3}\right)$.

On obtient les résultats suivants :

- Si $\left|\varphi^{\prime}-\varphi_{\mathrm{P}}^{\prime}\right|>\pi / 2$ surface cachée nulle;

- Si $\left|\theta^{\prime}-\theta_{\mathrm{P}}^{\prime}\right|>\pi / 2$ surface cachée nulle;

$-\operatorname{Si}\left\{\left|\varphi^{\prime}-\varphi_{\mathrm{P}}^{\prime}\right|<\pi / 2\right.$ et $\left\{\left|\theta^{\prime}-\theta_{\mathrm{P}}^{\prime}\right|<\pi / 2\right.$

$$
\begin{aligned}
& L_{\mathrm{c}}=\operatorname{Max}\left[0, d\left(1+\cos \varphi_{\mathrm{n}}^{\prime}+\operatorname{tg} \varphi_{\mathrm{P}}^{\prime} \sin \varphi_{\mathrm{n}}^{\prime}\right)-s \cos \theta^{\prime} \frac{\sin \left|\varphi^{\prime}-\varphi_{\mathrm{P}}^{\prime}\right|}{\cos \varphi_{\mathrm{P}}^{\prime}}\right], \\
& H_{\mathrm{c}}=\operatorname{Max}\left[0, h\left(1+\cos \theta_{\mathrm{n}}^{\prime}+\operatorname{tg} \theta_{\mathrm{P}}^{\prime} \sin \theta_{\mathrm{n}}^{\prime}\right)-s \cos \varphi^{\prime} \frac{\sin \left|\theta^{\prime}-\theta_{\mathrm{P}}^{\prime}\right|}{\cos \theta_{\mathrm{P}}^{\prime}}\right] .
\end{aligned}
$$

L'aire de surface cachée a pour expression $L_{\mathrm{c}} H_{\mathrm{c}}, d$ et $h$ étant respectivement la demi-largeur et la demihauteur de l'héliostat.

Ayant effectué ces calculs pour chaque paire d'héliostats avec comme vecteurs de projection $\mathbf{i}$ et $\mathbf{r}$, on superpose la surface ombrée et la surface masquée en supposant qu'en général elles ne se recouvrent pas. De même, en vérifiant qu'une partie de la surface de l'héliostat reste efficace, on calcule la surface totale de l'héliostat $\mathcal{H}$ cachée par tous les autres héliostats par simple sommation. On obtient ainsi une majoration assez fine de l'effet d'ombre sur $\mathscr{H}$ à un instant.

3. 3 CALCUL DES DENSITÉS DE FLUX SUR UNE CIBLE. - La cible, généralement supposée plane et rectangulaire est divisée suivant un maillage régulier. Pour chaque héliostat, on calcule tout d'abord la contribution à la densité de flux en chaque point du maillage sans tenir compte de l'effet d'ombre.'On calcule ensuite la surface totale de cet héliostat ombrée ou masquée par les autres miroirs. Le taux de perte par effet d'ombres est supposé s'appliquer uniformément sur la surface du miroir. Il permet de pondérer la contribution de cet héliostat à la densité de flux en chaque point.
A titre d'exemple, on a calculé les densités de flux sur le plan d'ouverture de la chaudière à cavité de la centrale THEMIS. Le champ d'héliostats de cette centrale comprend 201 héliostats. Il a été modélisé à partir des données EdF-REAM [6]. L'instant représenté sur la figure 6 correspond aux données solaires d'un 30 décembre à $10 \mathrm{~h}$ du matin :

$$
\begin{aligned}
E_{\mathrm{n}} & \simeq 0,769 \mathrm{~kW} \cdot \mathrm{m}^{-2} \\
\theta_{\mathrm{i}} & \simeq 0,643 \mathrm{rad} . \\
\varphi_{\mathrm{i}} & \simeq 0,673 \mathrm{rad} .
\end{aligned}
$$

Pour représenter les imprécisions de pointage, on a effectué un tirage aléatoire d'angles de dépointage uniformément répartis entre 0 et $3 \mathrm{mrad}$. sur le rayon réfléchi, pour des directions de dépointage aléatoires. En ce qui concerne les défauts des surfaces optiques, on a utilisé la valeur moyenne $\bar{\delta}=0,5 \mathrm{mrad}$. pour tous les héliostats. Les rayons de courbure des héliostats ont été supposés vérifier de façon approchée la relation $R=2 \mathrm{HZ}$. L'obtention de la carte de flux de la figure 6 a nécessité environ $30 \mathrm{~s}$ de temps de calcul sur l'IBM 370/168 du CIRCE-CNRS.

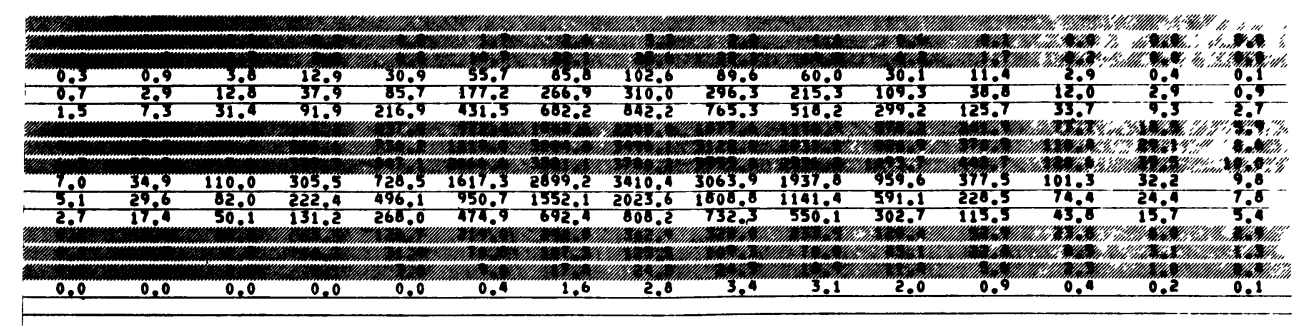

Fig. 6. - Carte de flux sur la cible.

[Map of flux on the target.] 
4. Conclusion. - Malgré les hypothèses simplificatrices utilisées pour résoudre les intégrales de densités de flux et pour obtenir les expressions des effets d'ombre entre héliostats, l'approche analytique proposée pour la modélisation et la simulation d'un champ d'héliostats présente des avantages considérables liés à la rapidité des calculs sur ordinateur et au caractère explicite des relations entre les variables. Elle permet en outre de simuler directement des politiques de commande de la centrale solaire par dépointage des héliostats de certaines zones (procé- dures d'urgence par exemple). Les hypothèses retenues sont raisonnables dans la mesure où les cartes de flux sont plus sensibles aux imprécisions de pointage qu'aux arrondis de calcul. La confrontation des résultats numériques obtenus par les méthodes analytiques que nous proposons avec les expériences qui seront effectuées sur la centrale THEMIS permettront l'identification des paramètres figurant dans le modèle d'ensoleillement et des lois probabilistes des erreurs de pointage.

\section{Bibliographie}

[1] Carraud, P., Giraud, A., Evaluation et Synthèse d'un Système de Concentration pour une Centrale Electrosolaire de $10 \mathrm{MW}$ électriques, Revue Phys. Appl. 13 (1978) 135-144.

[2] Biggs, F. et Vittitoe, C. N., The HELIOS Model for the Optical Behavior of Reflecting Solar Concentrators. Sandia Lab. Em. Report SAND 76-0347.

[3] Hennet, J. C., Méthodologie d'Evaluation et de Conception de Champs d'Héliostats Focalisants. Thèse de Doctorat d'Etat, Toulouse 1982.

[4] Hughes, R. O., Effects of Tracking Errors on the Performance of Point Focusing Solar Collectors, Solar Energy 24 (1980) 83-92.

[5] Lipps, F. W., Four Different Views of the Heliostat Flux Density Integral, Solar Energy 18 (1976) 555560 .
[6] Pharabod, F., Note Technique EdF-REAM E 12, janvier 1980.

[7] Projet THEM., EdF-CNRS, février 1977.

[8] Walzel, M. D., Lipps, F. W., Vant-Hull, L. L., A Solar Flux Density Calculation for a Solar Tower Concentration Using a Two Dimensional Hermite Function Expansion, Solar Energy 19 (1977) 239-253.

[9] Mersier, C., Les Centrales Electrosolaires à Tour. Optimisation du Champ de Réflecteurs et Application à l'Effet de Taille du champ, Revue Phys. Appl. 14 (1979).

[10] Courrege, P., Une Evaluation de l'Aire Efficace d'un Champ de Miroirs en terme de Répartition de Verre installé, Colloque International Energie Solaire, Toulouse, 1976. 